\title{
Converged Service Provision in Multidomain Environments for the Future Internet
}

\author{
SERGIO GONZALEZ-MIRANDA, TOMAS ROBLES, AUGUSTO MORALES, RAMÓN ALCARRIA, \\ EDUARDO PICO \\ Universidad Politécnica de Madrid, \\ Dpto. Ing. Sistemas Telemáticos (DIT), ETSI Telecomunicación (ETSIT), \\ Avenida Complutense s/n, Ciudad Universitaria, E-28040 Madrid (España), Office B-213 \\ *Authors: miranda@dit.upm.es, trobles@dit.upm.es, amorales@dit.upm.es, ralcarria@dit.upm.es, \\ eduardo_pico@hotmail.com
}

Future Internet requires a service provision that guarantees the adequate control of user related profiles and information. Current service enabler architectures provide attracting tool boxes to compose and provide advanced services in converged environments.

Keywords: Future Internet, IP Multimedia Subsystem, OMA, Service enablers, IPTV,

\section{IMS, NGN, ... FUTURE INTERNET}

IP Multimedia Subsystem (IMS) [2] is a set of specifications that describes the Next Generation Network (NGN) [1] architecture for implementing IP-based telephony and multimedia services. IMS defines a complete architecture and framework that enables the convergence of voice, video, data and mobile network technology over an IP-based infrastructure. It fills the gap between the two most successful communication paradigms, cellular and Internet technology. The vision for IMS is to provide cellular access to all the services that the Internet now provides. For example, by means of IMS a subscriber can use its $3 \mathrm{G}$ handheld device to surf the Web, play an online games or join videoconferences from anywhere. IMS was initially defined by the 3rd Generation Partnership Project (3GPP), which is a collaboration agreement among a number of telecommunications standards bodies, as part of their standardization work for supporting GSM networks and radio technology evolution. IMS was first introduced in 3GPP Release 5, where the "Session Initiation Protocol" (SIP), defined by the Internet Engineering Task Force (IETF), was chosen as the main signaling protocol for IMS. It has been further enhanced in Releases 6 and 7 of $3 \mathrm{GPP}$ to include additional features like presence and group management, interworking with WLAN and CS based systems (3GPP R6), and Fixed Broadband access (3GPP R7, by working together with TISPAN R1).

The IMS architecture is a unified architecture that supports a wide range of services enabled by the flexibility of SIP. The IMS architecture can support multiple application servers providing traditional telephony services and non-telephony services such as Instant Messaging (IM), Push-ToTalk (PTT), video streaming, multimedia messaging, etc.

IP Multimedia Subsystem (IMS) is on the way to becoming deployed for both mobile and fixed environments. It is hence necessary to have a way of unifying the different mobile and fixed identities that the user has. Managing user identities and group memberships information in today's connected systems is a crucial issue.

IPTV, Presence, Messaging, Chatting,... etcetera, represent an important set of multimedia services demanding the management of personalized, interactive and collaborative information in order to accelerate their integration into new converged services. 


\section{OMA SERVICE ENABLERS}

The Open Mobile Alliance (OMA) has specified market driven mobile service enablers that ensure service interoperability across devices, geographies, service providers, operators, and networks, while allowing businesses to compete through innovation and differentiation.

One of the roles of OMA is the specification of OMA enablers and how they use the interfaces of IMS. OMA has already developed several service enablers that use IMS functions and reference points, i.e. Push to talk Over Cellular (PoC), Presence Simple and SIP Push.

In tradition, service implementations have been done by integrating different components vertically and perservice. Reutilization of components has not been considered due to the lack of standards. This silo approach impedes the rapid deployment for new services and raises costs, common functions are duplicated among services, etcetera...

The OMA service enablers provides service environment with interoperable components leveraging the interaction between components and applications developed by different providers. This approach reduces deployment efforts by means of reusing common function components and allows interoperability across a wide variety of environments in a consistent manner.

In order to support the development of applications from different service providers, OMA has specified the OMA Service Environment (OSE).

It is a flexible and extensible architecture where service enablers perform their potentials always pursuing the reutilization of components and avoiding the reinvention of wheels.

OSE comprises: OMA enablers, enabler components and the interactions between them.

\section{CONVERGED SERVICE REALIZED}

Nowadays, TV content demand overwhelms the capacity of providing such service. This concept is reflected in the high volume of sales of HD tv receptors and the lack of availability of HD content in several zones around the globe.
Television content delivered via IP through network infrastructures is known as IPTv service. Tipically this service is supplied from a closed provider network infrastructure (private IP-based network) while, another competitor, Internet Television content service is delivered over the public Internet.

Because IPTV makes use of IP as a delivery mechanism, IP can be used to deliver various types of content over both the Internet and private IP-based networks. IPTv content can range in scope from music videos to television shows, full feature movies, rock concerts, and a variety of special events, such as boxing matches, football games, or even Broadway musicals.

Therefore, the concept of IPTV opens the market to a very wide range of both existing and potential activities.

Some of those activities could include downloading a movie or music video via the Internet for viewing now or at later date or subscribing to a television service that will be delivered to a homeowner via the installation of a private network that will provide the delivery of television content through the use of the IP. Thus, the term IPTV does not restrict content to that provided by broadcast television nor does it imply that delivery of content has to occur over the Internet. As we will note both later in this chapter as well as in other chapters throughout this book, IPTV represents a broad term used to reference the delivery of a wide variety of video content using the IP as a mechanism for transporting content.

\section{SERVICE CONTRIBUTIONS}

\section{Converged services architecture.}

IP Multimedia Subsystem (IMS) allows operators to provide users with converged and advanced services. Convenience for users and ease of management deriving into cost savings for operators motivate the creation of a converged service architecture where the same application layer infrastructure can be used to develop and deploy new and rich content services opening this walled-garden to third parties. The use of SIP as a signalling protocol standardizes the interfaces used by applications toward the core network and allows data sharing among multiple IMS applications. 
Nevertheless, this convergence concept cannot be realised before some serious problems are solved: Configuration storage, identity management, user preferences, etcetera.

\section{Integrated 3G-IPTv group management model}

The service-specific Application Server along with IMS service delivery mechanisms enable delivery of IPTv content and many other types of applications to subscribers.

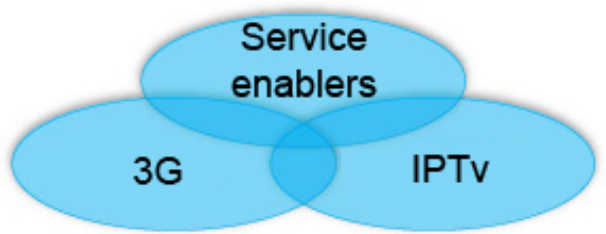

Service subscribers can access network's services, in this case, IPTV content, from any IP access network, including the public Internet and this content can be enriched with specific service enablers, e.g.: lists management. 3G and IPTv are merged into a service composition platform with standardized modules that perform specific functions at the request of these or other applications.

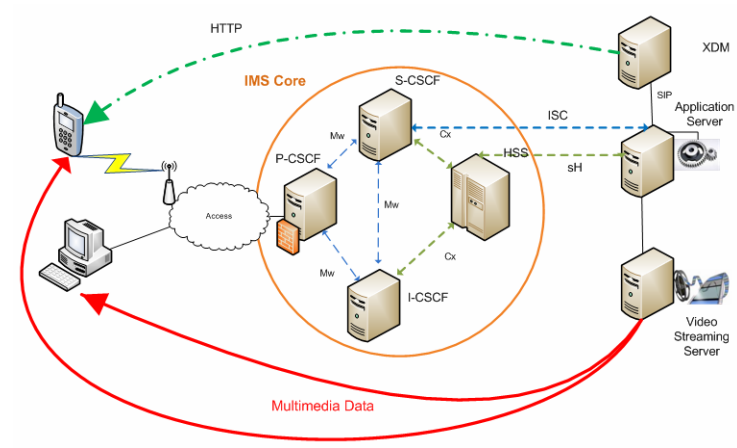

To comply with this purpose, a modular application engine is required to support the specialized complex technologies and standards needed in the provision of such a demanding realtime communication application and to ease the exchange and support of specialized and customized applications.

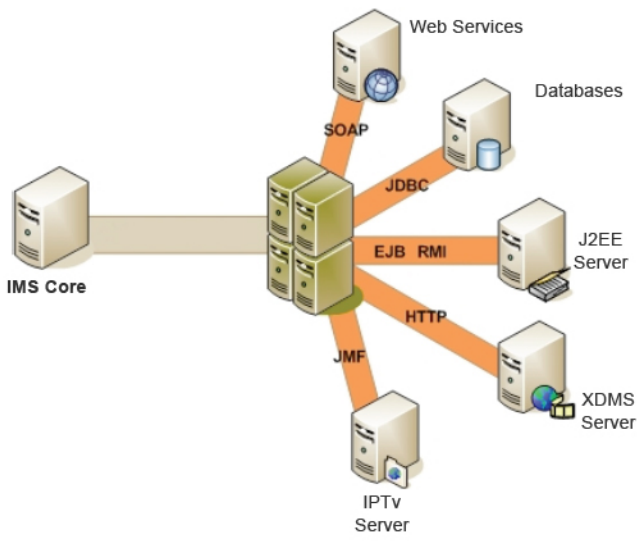

These application engines provide specialized functions needed by client applications and satisfy specific needs and requirements from the service. This approach helps in the creation of a complete solution for IPTv specific needs and requirements along with the control of access policy management.

The Session Control layer routes the call signaling and tells the transport layer what traffic to allow, and generates different types of information useful for the network. The CSCF is properly configured to perform and redirect incoming request to the adequate service provider (Application server).

The IMS Service/Application layer plays a crucial role in this context as it provides the infrastructure for the provision and management of services. It contains the logic software modules that are triggered according to service requests.

\section{SERVICE CASES}

Service cases such as: IPTv content management for $3 \mathrm{G}$ users and comment sharing in IPTV sessions shall be further described as our testbed gets more consolidated. It shall be enriched with more service applications.

\section{FUNDING}

This work is part of the MOBICOME project whose activities are carried out within the scope of the EUREKA program funding. The MOBICOME Consortium includes: Telenor, Linus, Oslo University College, HyC Soluciones de video y Comunicaciones, Universidad Politechnica de Madrid, TIWS, Telefonica Investigacion y Desarrollo S.A, HuaWei, Blekinge Institute of Technology, Ubisafe, HiQ. 


\section{REFERENCES}

[1] IMS as NGN Platform http://www.enterpriseireland.com/NR/rdonlyres/B93C82AE-1D154A7E-B63B-

713A98729D34/0/ThomasMagedanz.pdf [2] Wikipedia IP Multimedia Subsystem http://en.wikipedia.org/wiki/IP_Multimedia_Subs ystem [3] OMA

URL:http://www.openmobilealliance.com/ [4] OMA Presence SIMPLE V1.01

http://www.openmobilealliance.org/release_progr am/Presence_simple_v1_0_1.html

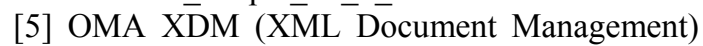
Shared

[6] Open Mobile Alliance, OMA Service Environment, 2007

[7] Open Mobile Alliance, OMA Utilization of IMS capabilities, 2005

[8] Open Mobile Alliance, Enabler Release Definition for IMS in OMA, 2005

[9] Stéphane H. Maes, "Service Delivery Platforms as IT Realization of OMA Service Environment: Service Oriented Architectures for Telecommunications". IEEE WCNC 2007 proceedings.

[10] Camarillo G., Garcia-Martin M., "The 3G IP Multimedia Subsystem (IMS), Merging the Internet and the Cellular Worlds", Second Edition. [11] Chakraborty S, Peisa J., Frankkila T., Synnergren. P., "IMS Multimedia Telephony over Cellular systems". 2007, ISBN 978-0-470-058558

[12] Poikselkä M., Mayer G., Khartabil H., Niemi A., "The IMS: IP Multimedia Concepts and Services", Second Edition, 2006, John Wiley \& Sons, Ltd. ISBN: 0-470-01906-9

[13] Van de Velde T., "Value added services for next generation networks", ISBN 978-0-84937318-3 [10] Schmidt M., Wilde A., Schülke A., Costa H., "IMS Interoperability and Conformance Aspects”, IEEE Communications Magazine, 2007 [14] Brenner M., Unmehopa M., , "The Open Mobile Alliance, Delivering Service Enablers for Next-Generation Applications", Wiley, 2007 